\title{
Serumcholinesterase und Myristoylcholinesterase der Leber bei Patienten mit Cholelithiasis
}

\author{
Von \\ E. MAIBACH \\ Aus dem Privatlaboratorium Dr. med. Maibach, Bern
}

(Der Schriftleitung zugegangen am 19. September 1963)

\begin{abstract}
Durch gleichzeitige Untersuchung der Serumcholinesterase durch den ACHOLEST-Streifentest und des Cholinesterasegehaltes der Leberzellen durch histochemische Methoden ließ sich bei 32 Patienten, die wegen Cholelithiasis laparatomiert werden mußten, feststellen, daß eine direkte Parallelität der Serumcholinesteraseaktivität und des Gehaltes der Leber an Cholinesterase besteht. Die Bestimmung der Serumcholinesterase ergibt somit ein deutliches Zustandsbild vom Cholinesterasegehalt der Leber.
\end{abstract}

A simultaneous study of serum cholinesterase by the ACHOLEST-strip test and the cholinesterase content of liver cells by histochemical methods was possible on 32 patients who had undergone laparatomy for cholelithiasis. Serum cholinesterase activity and the cholinesterase content of the liver were shown to be directly parallel. Thus the determination of serum cholinesterase gives a plain indication of the level of cholinesterase in the liver.

In sehr zahlreichen Arbeiten (1, 2, 3, 4) wurde auf einen Zusammenhang zwischen dem Gehalt des Serums an Cholinesterase („,SCHE“) und hepatozellulären Erkrankungen hingewiesen. Hierbei schien ein Absinken der SCHE-Aktivität bei zunehmendem Leberschaden die Regel zu bilden. Obgleich FIEsSINGer (5) einen Fall erwähnt, in dem bei einer atrophischen Lebercirrhose ein erhöhter SCHE-Wert gefunden wurde, und STREHLER und Mitarbeiter (1) darauf hinweisen, daß Fettlebern und auch akute Hepatitiden (als Reizzustand?) einen erhöhten SCHE-Gehalt aufweisen können, betonen die übrigen Autoren, daß im Rahmen mit anderen Leberfunktionsproben die Bestimmung der SCHE einen wichtigen diagnostischen Hinweis bilden kann bei der Beurteilung des Verlaufes von Leberkrankheiten. Dieselben Autoren erwähnen, daß eine leichte, bioptisch diagnostizierte Fibrosis der Leber keine pathologischen Leberfunktionsproben und auch normale SCHE-Werte ergaben. MANN und Mitarbeiter (6) fanden bei Verfettung der Leber ebenfalls normale SCHE-Aktivität. Normale SCHE-Konzentration bei gutartigem Gallengangverschluß oder bei Cholelithiasis beobachteten Fiessinger (5), Passouant (7) und Wissmer (8), während ANTOPOL (9) bei Gallengangserkrankungen ein Absinken der SCHE feststellte. Daß bei malignem Verschluß der Gallenwege die SCHE-Aktivität vermindert ist, berichteten ANTOpor (9), FiEssínger (5), Wissmer (8) und MANN (6). Enniedrigte Werte der SCHE bei Stauungsleber beobachtete DuLCE (10), ein Befund, den Orellana (11) auch bei der Wilsonschen Erkrankung erhob.

$\mathrm{DaB}$ die Bestimmung der SCHE-Aktivität nicht allein zur Labordiagnose einer Lebererkrankung herangezogen werden darf, betonen Fressinger (5), Vogt (2) und MANN (6). Ferner muß in Betracht gezogen werden, daß Veränderungen der SCHE-Werte auch bei anderen
Krankheiten, unabhängig von einem eventuellen Leberschaden, vorkommen $(9,12,13,14,10,5,2,4)$. Hierbei spielen insbesondere eine Rolle der Diabetes mellitus, arthritische Schübe, hochgradige Anämien und das nephrotische Syndrom. Zudem muß in Betracht gezogen werden, daß große individuelle Schwankungen bestehen, während die Normalwerte innerhalb eines Individuums große Konstanz aufweisen $(15,16,13,14$, 1). Außerdem berichtet VAHLQUist (15), daß weder anstrengende Muskelarbeit noch nervöse Anspannung die SCHE-Werte zu beeinflussen vermochten.

Im Gegensatz zu den zahlreichen physiologisch-chemischen Arbeiten sind die bistochemischen eher spärlich. Die Leber wurde am eingehendsten untersucht von GEREBTZOFF (17), der die Acetylthiocholinmethode von KoELLE (18) in einer Abwandlung verwendete. Er beobachtete hierbei starke, tier- und individuellspezifische Variationen des Enzymgehaltes der Leber, wobei aber innerhalb eines Individuums der Gehalt ziemlich konstant blieb. Das Ferment konnte in allen Leberabschnitten, vorwiegend aber zentrolobulär festgestellt werden.

Das Ziel der vorliegenden Arbeit ist, durch parallele und gleichzeitige Untersuchungen der SCHE-Aktivität und der Reaktion der unspezifischen Cholinesterase der Leber vermittelst der Myristoylcholintechnik eine eventuelle Abhängigkeit des Gehaltes des Serums an Cholinesterase vom Gehalt der Leber an demselben Ferment nachzuweisen.

\section{Methodik}

a) Material

Es wurden untersucht 32 Patienten (28 weiblich, 4 männlich) im Alter $z$ wischen 29 und $64 \mathrm{Jahren}$. Bei $31 \mathrm{Kranken}$ mußte die Laparatomie wegen Cholelithiasis, bei einem wegen Ulcus ventriculi vorgenommen werden. Bei einer Patientin lag ein Ikterus vor; bei den anderen Untersuchten lagen die Bilirubinwerte inner- 
halb der Norm. Bei der ikterischen Patientin waren zudem die alkalische Phosphatase leicht erhöht und der Takata +++ . Bei den übrigen Exploranden waren die Leberfunktionsproben normal. Keiner der Untersuchten war febril. Das Patientenmaterial war so ausgewählt, daß niemand an konkomittierenden Krankheiten litt, die auf den SCHE-Spiegel hätten einen Einfluß haben können.

\section{b) Bestimmung der SCHE}

Der SCHE-Titer wurde mittels der "Acholest"-Methode doppelt bestimmt. In 10 Fällen wurde eine dritte Kontrollbestimmung durch das Medizinisch-Chemische Institut Bern vorgenommen, wobei durchschnittlich etwas niedrigere Werte erhalten wurden. Das Blut wurde den nüchternen Patienten unmittelbar vor der Operation entnommen, sofort zentrifugiert und mit dem „Acholest"-Streifen mit der von SAILER und Mitarbeitern (3) und RICHTERICH (19) beschriebenen Methode sofort untersucht, wobei keine der von DuвACr (20) erwähnten Schwierigkeiten auftraten.

\section{c) Histocbemische Untersuchung}

Angewendet wurde die Myristoylcholin-Methode nach GomorI (21) modifiziert nach HARD und Mitarbeitern (22). Das bei der Operation entnommene Lebermaterial wurde unverzüglich nach der Gewinnung im Kryophor auf eine Dicke von $10 \mu$ geschnitten und anschließend in die Inkubationslösung, bestehend aus einem Gemisch von Myristoylcholin, Kobaltacetat und Veronal-AcetatPuffer $(\mathrm{pH}=7,6)$, gebracht. Die Schnitte wurden während 3 Stdn. bei $37^{\circ}$ inkubiert. Das nach der fermentativen Spaltung des Myristoylcholins an die höhere Fettsäure gebundene Kobalt wuirde mittels $\mathrm{H}_{2} \mathrm{~S}$ in Form eines feinen, schwarzen, körnigen Niederschlages zur Darstellung gebracht. Nach Gegenfärbung mit Eosin, Einbetten in Glyzeringelatine. $\mathrm{Da}$ durch das $\mathrm{H}_{2} \mathrm{~S}$ ebenfalls Haemosiderin gefärbt wird, wurden alle Schnitte, die bei der normalen pathologischen-anatomischen Untersuchung diesen Bestandteil aufwiesen, bei der Beurteilung verworfen. Entsprechende Kontrollschnitte wurden ohne Substrat inkubiert.

\section{d) Pathologisch-anatomische Untersuchung}

Die formolfixierten gefriergeschnittenen Leberpräparate wurden nach Färbung mit Haemalaun-Eosin, Sudan III und $\mathrm{H}_{2} \mathrm{~S}$ beurteilt. Die Untersuchungen wurden im pahtologisch-anatomischen Institut det Universität Bern (Prof. Dr. med. B. Walthard) vorgenommen.

\section{Ergebnisse}

a) Verhältnis zwischen SCHE-Aktivität und pathologisch-anatomischen Befunden

19 Untersuchte wiesen SCHE-Werte innerhalb der Norm (75-140 E) auf, 6 hatten erniedrigte, 7 erhöhte Werte.

Die Gruppe mit den erniedrigten SCHE-Werten wies pathologisch-anatomisch in einem Fall einen Ikterus der Leber auf (SCHE $56 \mathrm{E}$ ), in einem Fall diffuse Verfettung der Leber (SCHE $44 \mathrm{E}$ ), während die übrigen Fälle fleckförmige oder periphere Verfettung der Leber aufwiesen. Nur in einem Fall mit erniedrigtem SCHEWert $(40 \mathrm{E})$ wurde normales Lebergewebe diagnostiziert.

Die Gruppe mit normaler SCHE-Aktivität zeigte in 7 Fällen fettfreies Lebergewebe, während sonst meist fleckförmige Verfettung der Leber gefunden wurde. Diagnosen, welche derjenigen eines normalen Lebergewebes sehr nahe kommen. In einem Falle zeigte sich eine zentrale Leberverfettung, und nur in einer Untersuchung wurden vereinzelte Leberzellnekrosen, ver- stärkte Eosinophilie und Ballonierung der Leberzellen im Sinne eines leichten Leberzellschadens festgestellt, wobei der SCHE-Wert $105 \mathrm{E}$ betrug. In diesem Falle waren auch die üblichen Leberfunktionsproben negativ verlaufen.

Bei den Patienten mit erböbter SCHE-Tätigkeit wurde in 4 Fällen fettfreies Lebergewebe gefunden. Ein Patient wies fleckförmige, einer diffuse und einer zentrale Leberverfettung auf.

\section{b) Verhältnis $z$ wischen SCHE-Werten und Myristoylcholinaktivität in der Leber}

\section{Fälle mit normalem SCHE-Titer}

In allen Leberzellen der normal strukturierten Läppchen lassen sich feine schwarze Granula des an Myristinsäure gebundenen Kobaltsulfides feststellen. Die Körnchen sind in den peripheren Leberzellen meist etwas größer und reichlicher. Sie liegen meist in den zentralen $\mathrm{Ab}$ schnitten der Zelle, und man hat oft den Eindruck, daß sie den Gallenkapillaren anliegen. Daselbst sind hie und da die Kernmembranen und selten die Nukleolen schwarz angefärbt. Im intermediären Läppchenanteil sind etwas feinere und kleinere Körnchen in geringerer Anzahl vorhanden, während die zentralen Zellen am wenigsten granuläres Material enthalten. Dieses ist eher fein strukturiert. Hie und da sind die Granula der zentralen Zellen an das Lipofuscin angelagert. In vereinzelten Kupfferschen Sternzellen und Kapillarendothelien sind schwarze Körnchen vorhanden. Die Bindegewebszellen der Glissonschen Scheiden und der Leberkapsel sind frei von reagierendem Material. In den größeren Blutgefäßen sind im geronnenen Serum hie und da schwarze Granula feststellbar.

Die Kontrollschnitte zeigten keine Reaktion. - Die individuellen Schwankungen sind ziemlich deutlich ausgeprägt. Der Befund innerhalb eines Individuums jedoch bteibt durch die Schnittreihe hindurch ziemlich konstant. Die Reaktion bei Patienten mit niedrig-normalen SCHEWerten ist deutlich schwächer (weniger und feinere Granula) als bei denjenigen mit Mittelwerten, während Exploranden mit einer hochnormalen SCHE-Aktivität im Lebergewebe eine größere Reaktionsintensität (gröBere und mehr Granula) aufweisen.

\section{Fälle mit erböbtem SCHE-Titer}

In sämtlichen Zellen der normal gebauten Leberläppchen finden sich feine bis meist mittelgrobe oder selten grobe, schwarze Granula in ziemlich großer Menge. In den zentralen Leberzellen sind die feineren Körnchen meist vorherrschend. Sie sind meist diffus über die gesamte Zelle hin verteilt. Nicht selten sind sie an Fetttropfen oder an das Lipofuscin angelagert. Gegen den intermediären und peripheren Läppchenanteil hin nimmt die Reaktion an Intensität eher zu: die Zahl der Körnchen pro Zelle vermehrt sich, und die Granula werden gröber. Stellenweise sind die Zellen mit Reaktionsmaterial vollgestopft, wie es sich besonders deutlich bei demjenigen Fall mit einem SCHE-Titer von $350 \mathrm{E}$ zeigt. 
Im geronnenen Serum der mittleren und größeren Blutgefäße ist die Reaktion oft deutlich und stark positiv. In den Kupfferschen Sternzellen und in vereinzelten Kapillarendothelien lassen sich schwarze Granula feststellen. In den Glissonschen Scheiden und im Bindegewebe der Leberkapsel ist fleckweise ebenfalls schwarzes Reaktionsmaterial festzustellen; sehr wahrscheinlich handelt es sich hier um Verlagerungen, die während der Inkubation stattgefunden haben.

\section{Fälle mit erniedrigtem SCHE-Titer}

Im Gegensatz zu den beiden vorgehend beschriebenen Reaktionstypen sind in diesen Fällen nicht in allen Leberzellen schwarze Granula, die auf eine Cholinesteraseaktivität hindeuten, festzustellen. Im zentralen Läppchenanteil sind oft zahlreiche, oder bei ganz niederen SCHE-Werten die meisten Zellen ohne schwarze granuläre Einschlüsse, oder dann wechseln Stellen ohne Reaktion ab mit solchen, in denen die zentralen Leberzellen wenige oder vereinzelte feine bis selten mittelgroße schwarze Körnchen aufweisen. Die Reaktionsprodukte liegen eher in den zentralen Zellabschnitten. Die Zellen der intermediären Läppchenzone verhalten sich analog zu den zentralen Zellen. Erst in den peripheren Zellen kommen in etwas vermehrtem Ausmaß feine bis selten mittelgroße schwarze Reaktionsprodukte vor, die eher in den zentralen Zellabschnitten abgelagert sind. Thre Zahl variiert von Zelle zu Zelle sehr stark, und auch hier finden sich Zellen ohne schwarze Granula. Kupffer'sche Sternzellen, Kapillarendothelien sowie das Bindegewebe der Glissonschen Scheiden und der Leberkapsel zeigen keine Reaktionsprodukte. In dem Falle mit dem Ikterus der Leber (SCHE-Titer 56 E) kann in beinahe keiner Zelle Reaktionsmaterial gesehen werden. - In sämtlichen Fällen mit erniedrigter SCHE war die Reaktion der Myristoylcholinesterase der Leber deutlich schwächer als in denjenigen mit normaler oder erhöhter SCHE-Aktivität.

\section{Zusammenfassung der Befunde}

Die oben erhobenen Befunde lassen sich wie folgt in Tabelle 1 zusammenfassen:

\section{Diskussion}

Untersuchungen von GEREBTZOFF hatten mittels histochemischer Methoden große tier- und individuellspezifische Schwankungen der Reaktionsintensität der Cholinesterase der Leber aufgedeckt, Befunde, die physiologisch-chemisch am Serum in bezug auf die SCHE ebenfalls festgestellt worden waren. Ferner war von zahlreichen Autoren auf den hepatischen Ursprung der SCHE und deren Absinken bei Lebererkrankungen hingewiesen worden, so daß als Zusammenfassung all dieser Befunde Methoden zur Bestimmung der SCHE ausgearbeitet wurden, die dazu dienen sollten, als zusätzliche Leberfunktionsprobe im Laboratorium die Diagnosestellung hepatischer Erkrankungen zu erleichtern. Durch die Entwicklung eines Papierstreifens wurde die Methode auch für kleinere Laboratorien gut zugänglich gemacht.

In der vorliegenden Arbeit wird durch gleichzeitige Untersuchung des SCHE-Titers vermittels des Papierstreifentests („ACHOLEST") und der Myristoylcholinesterase der Leber dargelegt, daß eine Parallelität besteht zwischen der Aktivität der beiden Enzyme, bei denen es sich sehr wahrscheinlich ja um dasselbe Ferment, eine unspezifische Cholinesterase, handelt. Hierzu wurden Patienten verwendet, die sich wegen Cholelithiasis einer Laparatomie unterziehen mußten und die keine konkomittierenden Krankheiten aufwiesen. Zur besseren Beurteilbarkeit wurde das untersuchte Material unterteilt in drei Gruppen, nämlich eine mit normaler, eine mit erhöhter und eine mit pathologisch gesenkten SCHE-Werten. In bezug auf die pathologisch anatomische Untersuchung zeigte die Gruppe mit normaler SCHE-Tätigkeit meist normale Befunde mit Ausnahme eines Falles mit leichtem Leberzellschaden, in welchem aber auch die üblichen Leberfunktionsproben normal ausfielen. Die Reaktion der Myristoylcholinesterase der Leberzellen war in allen Fällen ziemlich intensiv, wobei das Ferment in Form eines schwarzen granulären Reaktionsproduktes in beinahe allen Zellen, vorzüglich in denjenigen der Läppchenperipherie, nachgewiesen werden konnte. Ausgehend von diesem "normalen“ Bild, das jedoch individuelle Schwankungen der Reak-

Tab. 1. Cholinesterase in Serum und Leber

\begin{tabular}{|c|c|c|c|c|c|c|c|}
\hline Leber & $\begin{array}{c}\text { SCHE*) } \\
\text { Myristoyl= } \\
\text { cholinesterase }\end{array}$ & $\begin{array}{l}\text { Żentrolobuläre } \\
\text { Zone }\end{array}$ & $\begin{array}{l}\text { Intermediäre } \\
\text { Zone }\end{array}$ & $\begin{array}{l}\text { Periphere } \\
\text { Zone }\end{array}$ & $\begin{array}{l}\text { Glissonsche } \\
\text { Scheiden }\end{array}$ & $\begin{array}{c}\text { Kupffersche } \\
\text { Sternzellen }+ \\
\text { Kapillarendothelien }\end{array}$ & $\begin{array}{c}\text { Gallengangs- } \\
\text { epithelien }\end{array}$ \\
\hline \multirow{3}{*}{ Serurn } & $\begin{array}{c}\text { SCHE } \\
\text { vermindert } \\
{[<75 \mathrm{E}]}\end{array}$ & $\begin{array}{c}\text { Granula } \\
\text { fehlend } \\
\text { oder + fein }\end{array}$ & $\begin{array}{c}\text { Granula } \\
\text { fehlend } \\
\text { oder + fein }\end{array}$ & $\begin{array}{l}\text { Granula } \\
\text { selten fehlend, } \\
\text { meist }+ \text {, fein }\end{array}$ & negativ & negativ & negativ \\
\hline & $\begin{array}{c}\text { SCHE } \\
\text { normal } \\
{[75-140 \mathrm{E}]}\end{array}$ & $+\rightarrow++$ & $+\rightarrow++$ & $++\rightarrow+++$ & negativ & $\begin{array}{l}\text { vereinzelt feine } \\
\text { Reaktionsgranula }\end{array}$ & negativ \\
\hline & $\begin{array}{c}\text { SCHE } \\
\text { vermehrt } \\
{[>140 \mathrm{E}]}\end{array}$ & $\begin{array}{l}+++\rightarrow \\
++++\end{array}$ & $\begin{array}{l}+++\rightarrow \\
++++\end{array}$ & +++ & $\begin{array}{c}\text { fleckenweise } \\
\text { Reaktionspro- } \\
\text { dukte. Diffusion? }\end{array}$ & $\begin{array}{c}\text { mäßig viele } \\
\text { feine Reaktions- } \\
\text { granula }\end{array}$ & negativ \\
\hline
\end{tabular}

*) $\mathrm{SCHE}=$ Serumcholinesterase. 
tionsintensität zeigte, wobei innerhalb eines Individuums die Reaktionsstärke ziemlich konstant blieb, ließen sich die beiden anderen Gruppen beurteilen. Patienten mit erböbten SCHE-Werten, deren Lebergewebe sich verhielt wie dasjenige der normalen Exploranden und bei denen keine pathologisch-anatomisch festlegbaren Leberschäden festgestellt werden konnten, zeigten eine sehr deutlich gesteigerte Aktivität der Myristoylcholinesterase-Aktivität in den Leberzellen, wobei es sogar zu Diffusionserscheinungen der Reaktionsprodukte in die Umgebung kam. Andererseits war bei Patienten mit vermindertem SCHE-Titer bereits in zwei Fällen das pathologisch-anatomische Bild der Leber bedeutungsvoll (Ikterus der Leber, diffuse Verfettung der Leber), wobei im ersten Fall auch Takata- und CadmiumReaktion pathologisch ausgefallen waren. - In allen Patienten dieser Gruppe war die Aktivität der Myristoylcholinesterase im Vergleich zu den Normalfällen deutlich herabgesetzt, und konnten Zellen oder ganze Zellbezirke beobachtet werden, in denen sich keine Reaktionsprodukte feststellen ließen.

Aus den obigen Untersuchungen läßt sich ohne weiteres der Schluß ziehen, daß eine Parallelität besteht zwischen dem SCHE-Titer und der Cholinesteraseaktivität der Leber, gemessen durch die MyristoylcholinesteraseBestimmung nach GoMORI. - Eine Übereinstimmung $z$ wischen dem pathologisch-anatomischen Bild der Leber und der SCHE-Aktivität besteht nicht; ebenso lassen sich die verschiedenen Typen von Leberverfettung in allen drei Gruppen von SCHE-Aktivität feststellen, so daß dieses pathologisch-anatomische Zustandsbild des Organes nicht aus dem Verhalten der SCHE diagnostiziert werden darf.

Es kann somit hervorgehoben werden, daß die SCHEAktivität bei geringfügigen, noch nicht als Leberschaden anzusprechenden pathologisch-anatomischen Leberveränderungen sich nicht $z u$ verändern braucht (aber sich verändern kann), währenddem sie jedoch einen deutlichen Hinweis auf den Gehalt der Leber an Cholinesterase darstellt. Bezeichnet man die Fähigkeit eine dem Organ zustehende Funktion -z z. B. Bildung oder Speicherung von Cholinesterase - nicht voll zu erfüllen, als einen Organschaden, so kann man definitionsgemäß eine Verminderung der Myristoylcholinreaktion als Hinweis auf eine Leberschädigung annehmen. $\mathrm{Da}$ aber laut den vorigen Befunden eine deutliche Parallelität $z$ wischen Myristoylcholinesterasereaktion dèr Leber und SCHEAktivität besteht, so darf eine Verminderung des SCHETiters füglich als ein Hinweis auf einen möglichen Leberschaden angesehen werden. $\overline{\mathrm{Da}}$ andererseits die durch den Papierstreifentest gewonnenen Werte und die daraus resultierende Gruppeneinteilung sich mit dem histochemisch an der Leber erhobenen Befund deckt, ist auszusagen, daß die Sensibilität des Tests groß genug ist, um ein Bild der SCHE-Tätigkeit und des dahinterliegenden Leberbildes $z u$ vermitteln.

\section{Literatur}

1. Strehler und Meyer, Helvet. med. acta 19, 555 (1952). - 2. Vogr, Helvet. med. acta 20, 356 (1953). - 3. SAILER und BrauNSTEINER, Klin. Wschr. 37, 986 (1959). - 4. Richterich, R., Praxis, Bern 24, 624 (1961). - 5. Fressinger, Glomaud und Carlotti, Bull. Acad. med. 128, 293 (1944). - 6. ManN, Mandel, Eichmann, Knowlton und Sborov, J. Laborat. Clin. Med., S. Louis 39, 543 (1952). - 7. Passouant und Benezech, Arch. mal. app. digest., Paris 37, 77 (1948). - 8. Wissmer, Gastroenterologia, Basel 78, 111 (1952). - 9. ANTopol, Schiefrin und Tuchman, Proc. Soc. exp. Biol. Med. 38, 363 (1938). 10. Dulce, H.-J., Zschr. inn. Med., Leipzig 4, 174 (1949). - 11. Orellana und Alcalde, J. Laborat. Clin. Med., S. Louis 36, 391
(1950). - 12. Mc. ArdLe, Quart. J. Med. 9, 107 (1940). 13. F FABE, Acta med. Scand. 64, 59 (1943). - 14. BRECHT, Dtsch. med. Wschr. 71, 445 (1946). - 15. VAhLQUist, Scand. Arch. Physiol. 72, 133 (1935). - 16. LACKey und SLAughtÉ, J. Laborat. Clin. Med., S. Louis 27, 640 (1942). - 17. Gerebtzoff, C. R. Séances Soc. Bịol. Filiales 148, 397 (1954). - 18. Koelle, J. Pharmacy Pharmacol. 103, 153 (1951). - 19. Richterich, R., Schweiz. med. Wschr. 92, 263 (1962). - 20. Dubach, Praxis, Bern 35, 870 (1962). - 21. Gomori, Proc. Soc. exp. Biol. Med. 68, 354 (1948). - 22. Hard und Peterson. Anat. Rec., Philadelphia 108, 57 (1950).
Dr. med. Ed. Maibach Bern (Schweiz) Könizstraße 3 